\title{
Evaluation of mechanical, morphological, and biodegradable properties of hybrid natural fiber polymer nanocomposites
}

\begin{abstract}
Hybrid natural fiber polymer nanocomposites were prepared using various natural fibers (kenaf, coir, and wood), polypropylene, and montmorillonite nanoclay through the hot compression method. The effects of fiber hybridization and nanoclay content on the physicomechanical and biodegradable properties of the synthesized composites were investigated. Fourier-transform infrared and scanning electron microscopic analyses indicated that the structure and surface morphology of composites were transformed after fiber hybridization and the subsequent nanoclay incorporation. X-ray diffraction pattern revealed that the percent crystallinity of hybrid nanocomposites significantly increased. Furthermore, the tensile strength and tensile modulus also significantly improved for the hybrid nanocomposites due to the addition of montmorillonite nanoclay. The biodegradability and water absorption tests were conducted. The results show that biodegradability of the nanocomposites decreased and water absorption increased due to the addition of montmorillonite nanoclay.
\end{abstract}

Keyword: Hybrid natural fiber polymer nanocomposites; Biodegradable properties; Physicomechanical 